\title{
ON HAUSDORFF DIMENSION OF RADIAL PROJECTIONS
}

\author{
BOCHEN LIU
}

Abstract. For any $x \in \mathbb{R}^{d}, d \geqslant 2$, denote $\pi^{x}: \mathbb{R}^{d} \backslash\{x\} \rightarrow S^{d-1}$ as the radial projection

$$
\pi^{x}(y)=\frac{y-x}{|y-x|}
$$

Given a Borel set $E \subset \mathbb{R}^{d}$, $\operatorname{dim}_{\mathcal{H}} E \leqslant d-1$, in this paper we investigate for how many $x \in \mathbb{R}^{d}$ the radial projection $\pi^{x}$ preserves the Hausdorff dimension of $E$, namely whether $\operatorname{dim}_{\mathcal{H}} \pi^{x}(E)=\operatorname{dim}_{\mathcal{H}} E$. We develop a general framework to link $\pi^{x}(E), x \in F$ and $\pi^{y}(F), y \in E$, for any Borel set $F \subset \mathbb{R}^{d}$. In particular, whether $\operatorname{dim}_{\mathcal{H}} \pi^{x}(E)=\operatorname{dim}_{\mathcal{H}} E$ for some $x \in F$ can be reduced to whether $F$ is visible from some $y \in E$ (i.e. $\mathcal{H}^{d-1}\left(\pi^{y}(F)\right)>0$ ). This allows us to apply Orponen's estimate on visibility to obtain

$$
\operatorname{dim}_{\mathcal{H}}\left\{x \in \mathbb{R}^{d}: \operatorname{dim}_{\mathcal{H}} \pi^{x}(E)<\operatorname{dim}_{\mathcal{H}} E\right\} \leqslant 2(d-1)-\operatorname{dim}_{\mathcal{H}} E,
$$

for any Borel set $E \subset \mathbb{R}^{d}, \operatorname{dim}_{\mathcal{H}} E \in(d-2, d-1]$. This improves the Peres-Schlag bound when $\operatorname{dim}_{\mathcal{H}} E \in\left(d-\frac{3}{2}, d-1\right]$, and it is optimal at the endpoint $\operatorname{dim}_{\mathcal{H}} E=d-1$.

\section{INTRODUCTION}

For any $x \in \mathbb{R}^{d}, d \geqslant 2$, denote $\pi^{x}: \mathbb{R}^{d} \backslash\{x\} \rightarrow S^{d-1}$ as the radial projection

$$
\pi^{x}(y)=\frac{y-x}{|y-x|}
$$

We say a Borel set $E \subset \mathbb{R}^{d}$ is

- visible from $x$, if $\mathcal{H}^{d-1}\left(\pi^{x}(E)\right)>0$, and

- invisible from $x$, if $\mathcal{H}^{d-1}\left(\pi^{x}(E)\right)=0$,

where $\mathcal{H}^{d-1}$ denotes the $(d-1)$-dimensional Hausdorff measure.

The study of visibility has a long history in geometry measure theory (see, for example, Section 6 in Mattila's survey [6]). It dates back to Marstrand's celebrated 1954 paper [5], where it is proved that given a Borel set $E \subset \mathbb{R}^{2}, 0<\mathcal{H}^{s}(E)<\infty$ for some $s>1$, then $E$ is visible from almost all $x \in \mathbb{R}^{2}$, and also from $\mathcal{H}^{s}$ almost all $x \in E$. Recently, due to Mattila-Orponen [8] and Orponen [9] [10], it is proved that given any Borel set $E \subset \mathbb{R}^{d}, \operatorname{dim}_{\mathcal{H}} E>d-1$, then

$$
\operatorname{dim}_{\mathcal{H}}\left\{x \in \mathbb{R}^{d}: \mathcal{H}^{d-1}\left(\pi^{x}(E)\right)=0\right\} \leqslant 2(d-1)-\operatorname{dim}_{\mathcal{H}} E .
$$

This dimensional exponent is sharp [9]: for any $\alpha \in(d-1, d]$, there exists a Borel set $E \subset \mathbb{R}^{d}, \operatorname{dim}_{\mathcal{H}} E=\alpha$, which is invisible from a set of Hausdorff dimension $2(d-1)-\alpha$.

When $0<\mathcal{H}^{d-1}(E)<\infty$, visibility depends on rectifiability. One can see, for example, Section 6 in Mattila's survey [6], more recent results in [14], [11], [1], and references therein.

2010 Mathematics Subject Classification. 28A75.

Key words and phrases. radial projection, visibility, Hausdorff dimension. 
For any $\omega \in S^{d-1}$, denote $P_{\omega}: \mathbb{R}^{d} \rightarrow \omega^{\perp}$ as the orthogonal projection. The wellknown Marstrand projection theorem states that: given a Borel set $E \subset \mathbb{R}^{2}$, then for almost all $\omega \in S^{1}$,

- $\left|P_{\omega}(E)\right|>0$, if $\operatorname{dim}_{\mathcal{H}} E>1$;

- $\operatorname{dim}_{\mathcal{H}} P_{\omega}(E)=\operatorname{dim}_{\mathcal{H}} E$, if $\operatorname{dim}_{\mathcal{H}} E \leqslant 1$.

Inspired by Marstrand's result, for any projection $\pi$ from a higher dimensional space $U$ to a lower dimensional space $V$, the following two questions are natural.

Q1: Given $E \subset U$, $\operatorname{dim}(E)>\operatorname{dim}(V)$, does $\pi(E)$ have positive density in $V$ ?

Q2: Given $E \subset U, \operatorname{dim}(E) \leqslant \operatorname{dim}(V)$, does $\pi(E)$ preserve the dimension of $E$ ?

For radial projections, Q1 has already been answered by (1.1). So it remains to consider Q2, namely given $\operatorname{dim}_{\mathcal{H}} E \leqslant d-1$ whether

$$
\operatorname{dim}_{\mathcal{H}} \pi^{x}(E)=\operatorname{dim}_{\mathcal{H}} E .
$$

There is very little known on Hausdorff dimension of radial projections. The PeresSchlag machinery [12] implies that when $\operatorname{dim}_{\mathcal{H}} E \leqslant d-1$,

$$
\begin{gathered}
\operatorname{dim}_{\mathcal{H}}\left\{x \in \mathbb{R}^{d}: \operatorname{dim}_{\mathcal{H}} \pi^{x}(E)<\tau\right\} \leqslant \tau+1, \forall \tau \in\left(\operatorname{dim}_{\mathcal{H}} E-1, \operatorname{dim}_{\mathcal{H}} E\right) ; \\
\operatorname{dim}_{\mathcal{H}} \pi^{x}(E) \geqslant \operatorname{dim}_{\mathcal{H}} E-1, \forall x \in \mathbb{R}^{d} .
\end{gathered}
$$

In particular, (1.2) implies

$$
\operatorname{dim}_{\mathcal{H}}\left\{x \in \mathbb{R}^{d}: \operatorname{dim}_{\mathcal{H}} \pi^{x}(E)<\operatorname{dim}_{\mathcal{H}} E\right\} \leqslant \operatorname{dim}_{\mathcal{H}} E+1 .
$$

Given $E \subset \mathbb{R}^{2}$ which does not lie in a line, Orponen [10] showed

$$
\operatorname{dim}_{\mathcal{H}}\left\{x \in \mathbb{R}^{2}: \operatorname{dim}_{\mathcal{H}} \pi^{x}(E)<\frac{\operatorname{dim}_{\mathcal{H}} E}{2}\right\}=0
$$

None of these results seems to be sharp. The Peres-Schlag bound is trivial when $\tau=$ $\operatorname{dim}_{\mathcal{H}} E=d-1$, and Orponen's bound still has a gap to our expectation $\operatorname{dim}_{\mathcal{H}} \pi^{x}(E)=$ $\operatorname{dim}_{\mathcal{H}} E$.

In this paper we introduce a new framework to study radial projections. We discover a connection between $\pi^{x}(E), x \in F$ and $\pi^{y}(F), y \in E$, for any Borel set $F \subset \mathbb{R}^{d}$. Roughly speaking whether $\operatorname{dim}_{\mathcal{H}} \pi^{x}(E) \geqslant \tau$ for some $x \in F$ can be reduced to whether $\operatorname{dim}_{\mathcal{H}} \pi^{y}(F) \geqslant d-1+\tau-\operatorname{dim}_{\mathcal{H}} E$ for some $y \in E$. In particular, whether $\operatorname{dim}_{\mathcal{H}} \pi^{x}(E)=$ $\operatorname{dim}_{\mathcal{H}} E$ for some $x \in F$ can be reduced to whether $\mathcal{H}^{d-1}\left(\pi^{y}(F)\right)>0$ for some $y \in E$. This allows us to apply Orponen's estimate [10] in the proof of (1.1) to obtain the following.

Theorem 1.1. Given a Borel set $E \subset \mathbb{R}^{d}, d \geqslant 2$, $\operatorname{dim}_{\mathcal{H}} E \in(d-2, d-1]$, then

$$
\operatorname{dim}_{\mathcal{H}}\left\{x \in \mathbb{R}^{d}: \operatorname{dim}_{\mathcal{H}} \pi^{x}(E)<\operatorname{dim}_{\mathcal{H}} E\right\} \leqslant 2(d-1)-\operatorname{dim}_{\mathcal{H}} E .
$$

Equivalently, given Borel sets $E, F \subset \mathbb{R}^{d}, \operatorname{dim}_{\mathcal{H}} E \in(d-2, d-1], \operatorname{dim}_{\mathcal{H}} F>2(d-$ 1) $-\operatorname{dim}_{\mathcal{H}} E$, then there exists $y \in F$ such that $\operatorname{dim}_{\mathcal{H}} \pi^{x}(E)=\operatorname{dim}_{\mathcal{H}} E$.

Theorem 1.1 improves (1.4) when $\operatorname{dim}_{\mathcal{H}} E \in\left(d-\frac{3}{2}, d-1\right]$. It is optimal at the endpoint $\operatorname{dim}_{\mathcal{H}} E=d-1$ (e.g. a hyperplane), but I don't think it is generally sharp. It would be nice to show given $\operatorname{dim}_{\mathcal{H}} E \leqslant d-1$,

$$
\operatorname{dim}_{\mathcal{H}}\left\{x \in \mathbb{R}^{d}: \operatorname{dim}_{\mathcal{H}} \pi^{x}(E)<\operatorname{dim}_{\mathcal{H}} E\right\} \leqslant d-1,
$$


or any counterexample. More generally I am wondering if the following holds. Notice it is always better than the Peres-Schlag bound (1.4).

Conjecture 1.2. Given a Borel set $E \subset \mathbb{R}^{d}, d \geqslant 2, \operatorname{dim}_{\mathcal{H}} E \in(k-1, k], k=1,2, \ldots, d-$ 1 , then

$$
\operatorname{dim}_{\mathcal{H}}\left\{x \in \mathbb{R}^{d}: \operatorname{dim}_{\mathcal{H}} \pi^{x}(E)<\operatorname{dim}_{\mathcal{H}} E\right\} \leqslant k
$$

One may also wonder if anything new follows by plugging (1.2), (1.3), (1.5) into our framework. Unfortunately, the answer is negative. However, we still expect that our method would help for improvement in the future. See Section 5 for discussion.

1.1. Radial projection and the distance problem. Some ideas in this paper are inspired by recent work on the Falconer distance conjecture, which states that when the Hausdorff dimension of $E \subset \mathbb{R}^{d}, d \geqslant 2$ is large enough, the set of distances

$$
\Delta(E)=\{|y-x|: x, y \in E\}
$$

must have positive Lebesgue measure. In a recent paper of Guth, Iosevich, Ou and Wang [2], they group wave packets into good/bad families and work on them separately. Although in this paper we don't work with wave packets, the idea of good/bad tubes still helps. Their estimate also works on the Hausdorff dimension of distance sets (see [4], [13]).

On the other hand, in recent work on distances [3] [2], a radial projection estimate due to Orponen [10] plays an important role. Therefore it has its own interest to see that these two problems interact with each other.

Notation. $X \lesssim Y$ means $X \leqslant C Y$ for some constant $C>0 . X \approx Y$ means $X \lesssim Y$ and $Y \lesssim X$.

Throughout this paper $\phi \in C_{0}^{\infty}\left(\mathbb{R}^{d}\right), \phi \geqslant 0, \int \phi=1$, and $\phi \geqslant 1$ on $B\left(0, \frac{1}{2}\right)$. Denote $\phi_{\delta}(\cdot)=\frac{1}{\delta^{d}} \phi(\dot{\bar{\delta}})$ and $\mu^{\delta}=\mu * \phi_{\delta}$ for any compactly supported Borel measure $\mu$ on $\mathbb{R}^{d}$.

$\widehat{f}(\xi):=\int e^{-2 \pi i x \cdot \xi} f(x) d x$ denotes the Fourier transform.

For any finite Borel measure $\mu$ on $\mathbb{R}^{d}$, denote $\|\mu\|=\mu\left(\mathbb{R}^{d}\right)$.

Acknowledgments. I would like to thank Tuomas Orponen for comments, and reminding me of adding Conjecture 1.2 into literature.

\section{Preliminaries}

2.1. Frostman measures. Suppose $E \subset \mathbb{R}^{d}$ is a Borel set. It is well known that for any $s_{E}<\operatorname{dim}_{\mathcal{H}} E$ there exists a finite Borel measure $\mu_{E}$ supported on $E$, called a Frostman measure, such that

$$
\mu_{E}(B(x, r)) \leqslant r^{s_{E}}, \forall x \in \mathbb{R}^{d}, r>0 .
$$

As a consequence, for any $s<s_{E}$ the energy integral

$$
I_{s}\left(\mu_{E}\right)=\iint|x-y|^{-s} d \mu_{E}(x) d \mu_{E}(y)=c_{d} \int\left|\widehat{\mu_{E}}(\xi)\right|^{2}|\xi|^{-d+s} d \xi<\infty .
$$

For more information about Frostman measures and energy integrals, see, for example, [7], Section 2.5, 3.5. 
2.2. Hausdorff dimension of radial projections. We shall use the following criteria to determine Hausdorff dimension. It is standard in geometric measure theory. Throughout this paper $\delta_{k}=2^{-k}$.

Lemma 2.1. Given $E \subset \mathbb{R}^{d}, x \in \mathbb{R}^{d}$, and a finite Borel measure $\mu_{E}$ on $E$. Suppose there exist $\tau \in(0, d-1], K \in \mathbb{Z}_{+}, \beta>0$ such that

$$
\mu_{E}\left(\left\{y: \pi^{x}(y) \in D_{k}\right\}\right)<\delta_{k}^{\beta}
$$

for any

$$
D_{k} \in \Theta_{k}^{\tau}:=\left\{\bigcup_{j=1}^{M} \theta_{j}: M \leqslant \delta_{k}^{-\tau}, \theta_{j} \subset S^{d-1} \text { is a } \delta_{k}-c a p, j=1, \ldots, M\right\} .
$$

Then

$$
\operatorname{dim}_{\mathcal{H}} \pi^{x}(E) \geqslant \tau
$$

We give the proof for completeness.

Proof. If

$$
\operatorname{dim}_{\mathcal{H}} \pi^{x}(E)<\tau
$$

there exists $s \in\left(\operatorname{dim}_{\mathcal{H}} \pi^{x}(E), \tau\right)$ such that $\mathcal{H}^{s}\left(\pi^{x}(E)\right)=0$. By the definition of Hausdorff measure there exists an integer $N_{0}>0$ such that for any integer $N>N_{0}$, we can find a cover $\Theta$ of $\pi^{x}(E)$ consisting of finitely many caps of radius $<2^{-N}$, such that

$$
\sum_{\theta \in \Theta} \operatorname{diam}(\theta)^{s} \leqslant 1
$$

Denote

$$
\Theta_{k}=\left\{\theta \in \Theta: 2^{-k-1} \leqslant \operatorname{diam}(\theta)<2^{-k}\right\}, k=N, N+1, \ldots
$$

and

$$
D_{k}=\bigcup_{\theta \in \Theta_{k}} \theta
$$

We may assume $N>\frac{s}{\tau-s}$. Then

$$
\#\left(\Theta_{k}\right) \leqslant 2^{(k+1) s} \leqslant 2^{k \tau} .
$$

Since

$$
\pi^{x}(E) \subset \bigcup_{k \geqslant N} D_{k}
$$

and

$$
\mu_{E}\left(\left\{y \in E: \pi^{x}(y) \in \pi^{x}(E)\right\}\right)=\left\|\mu_{E}\right\|,
$$

it follows that there exists $k_{0} \geqslant 0$ such that

$$
\mu_{E}\left(\left\{y \in E: \pi^{x}(y) \in D_{k_{0}+N}\right\}\right)>\frac{\left\|\mu_{E}\right\|}{100\left(k_{0}+1\right)^{2}} .
$$

On the other hand, for any $N>K$, the assumption in the lemma implies

$$
\mu_{E}\left(\left\{y \in E: \pi^{x}(y) \in D_{k_{0}+N}\right\}\right)<2^{-\left(N+k_{0}\right) \beta},
$$

which is a contradiction when $N$ is large enough so that

$$
2^{-N \beta}<\inf _{k \geqslant 0} \frac{2^{k \beta}}{100(k+1)^{2}}\left\|\mu_{E}\right\| .
$$


3. From $\pi^{x}(E), x \in F$ то $\pi^{y}(F), y \in E$

Given Borel sets $E, F \subset \mathbb{R}^{d}$ and $0 \leqslant \tau \leqslant \operatorname{dim}_{\mathcal{H}} E$, in this section we investigate if there exists $x \in F$ such that $\operatorname{dim}_{\mathcal{H}} \pi^{x}(E) \geqslant \tau$. Without loss of generality we may assume both $E, F$ are compact, $\operatorname{dist}(E, F) \approx 1, \operatorname{dim}_{\mathcal{H}} E, \operatorname{dim}_{\mathcal{H}} F>0$ and $0<\tau<\operatorname{dim}_{\mathcal{H}} E$. Let $\mu_{E}, \mu_{F}$ be Frostman measures on $E, F$ satisfying (2.1) with $\tau<s_{E}<\operatorname{dim}_{\mathcal{H}} E$, $s_{F}<\operatorname{dim}_{\mathcal{H}} F$.

To show $\operatorname{dim}_{\mathcal{H}} \pi^{x}(E) \geqslant \tau$ for some $x \in F$, it suffices to show the condition in Lemma 2.1 is satisfied for $\mu_{F^{-}}$a.e. $x \in F$.

Let $\beta>0$ be a small number that will be specified later. Denote $F$ as the subset of $F$ consisting of points $x \in F$ such that

$$
\mu_{E}\left(\left\{y: \pi^{x}(y) \in D_{k}\right\}\right) \geqslant \delta_{k}^{\beta}
$$

for some $D_{k} \in \Theta_{k}^{\tau}$. In other words, $F$ consists of points $x \in F$ where the condition in Lemma 2.1 fails at scale $\delta_{k}$. Then

$$
\begin{aligned}
\delta_{k}^{\beta} \mu_{F}\left(F_{k}\right) & \leqslant \int_{F_{k}} \sup _{D_{k} \in \Theta_{k}^{\tau}} \mu_{E}\left(\left\{y: \pi^{x}(y) \in D_{k}\right\}\right) d \mu_{F}(x) \\
& \leqslant \int \sup _{D_{k} \in \Theta_{k}^{\tau}} \mu_{E}\left(\left\{y: \pi^{x}(y) \in D_{k}\right\}\right) d \mu_{F}(x) .
\end{aligned}
$$

If one could show that

$$
\int \sup _{D_{k} \in \Theta_{k}^{\tau}} \mu_{E}\left(\left\{y: \pi^{x}(y) \in D_{k}\right\}\right) d \mu_{F}(x) \lesssim \delta_{k}^{2 \beta},
$$

then $\mu_{F}\left(F_{k}\right)$ is summable in $k$. By the Borel-Cantelli Lemma, the condition in Lemma 2.1 is satisfied for $\mu_{F}$-a.e. $x \in F$, as desired.

It remains to show (3.2). For any $x, y \in \mathbb{R}^{d}, x \neq y$, denote $l_{x, y}$ as the line passing through $x, y$ and $T_{x, y}^{k}$ as the $\left(10 \delta_{k}\right)$-neighborhood of $l_{x, y}$. Take $s=d-1+\tau-s_{E}+4 \beta$, define

$$
\begin{gathered}
\operatorname{Good}_{k, s}=\left\{(x, y) \in \mathbb{R}^{d} \times \mathbb{R}^{d}: \mu_{F}\left(T_{x, y}^{k}\right)<\delta_{k}^{s}\right\}, \operatorname{Good}_{k, s}^{x}=\left\{y:(x, y) \in \operatorname{Good}_{k, s}\right\} ; \\
\operatorname{Bad}_{k, s}=\left\{(x, y) \in \mathbb{R}^{d} \times \mathbb{R}^{d}: \mu_{F}\left(T_{x, y}^{k}\right) \geqslant \delta_{k}^{s}\right\}, \operatorname{Bad}_{k, s}^{x}=\left\{y:(x, y) \in \operatorname{Bad}_{k, s}\right\},
\end{gathered}
$$

and denote

$$
\mu_{E, \operatorname{Good}_{k, s}^{x}}=\left.\mu_{E}\right|_{\operatorname{Good}_{k, s}^{x}}, \mu_{E, B_{k, s}^{x}}=\left.\mu_{E}\right|_{\operatorname{Bad}_{k, s}^{x}} .
$$

Under this notation, for each $x \in F$, we have $\mu_{E}=\mu_{E, \operatorname{Good}_{k, s}^{x}}+\mu_{E, \text { Bad }_{k, s}^{x}}$, where $\mu_{E, \operatorname{Good}_{k, s}^{x}}, \mu_{E, \text { Bad }_{k, s}^{x}}$ are both compactly supported Borel measures. Then the left hand side of (3.2) is bounded above by

$$
\begin{aligned}
& \int \sup _{D_{k} \in \Theta_{k}^{\tau}} \mu_{E, \operatorname{Good}_{k, s}^{x}}\left(\left\{y: \pi^{x}(y) \in D_{k}\right\}\right) d \mu_{F}(x)+\int \mu_{E, B a d_{k, s}^{x}}\left(\mathbb{R}^{d}\right) \mu_{F}(x) \\
= & \text { Good + Bad. }
\end{aligned}
$$

To move on, we need the following observation. For any $D_{k} \in \Theta_{k}^{\tau}$, denote $\widetilde{D_{k}}$ as the $\delta_{k}$-neighborhood of $D_{k}$. 
Lemma 3.1. For any Borel measure $\mu$ on $\mathbb{R}^{d}, D_{k} \in \Theta_{k}^{\tau}, x \in \mathbb{R}^{d}, \operatorname{dist}(x, \operatorname{supp}(\mu)) \gtrsim 1$, we have

$$
\mu\left(\left\{y: \pi^{x}(y) \in D_{k}\right\}\right) \lesssim \int_{\widetilde{D_{k}}} \pi_{*}^{x}\left(\mu^{\delta_{k}}\right)(\omega) d \omega
$$

Here the implicit constant only depends on $\operatorname{dist}(x, \operatorname{supp}(\mu))$.

Proof. Notice the right hand side equals

$$
\begin{aligned}
\int_{\pi^{x}(z) \in \widetilde{D_{k}}} \mu^{\delta_{k}}(z) d z & =\delta_{k}^{-d} \iint_{\pi^{x}(z) \in \widetilde{D_{k}}} \phi\left(\delta_{k}^{-1}(z-y)\right) d \mu(y) d z \\
& \geqslant \delta_{k}^{-d} \iint_{\pi^{x}(z) \in \widetilde{D_{k}},|y-z| \leqslant \frac{\delta_{k}}{2}} d z d \mu(y) .
\end{aligned}
$$

Fix $y$ and integrate $z$ first. Since $|x-y| \gtrsim 1$, we have $\pi^{x}\left(B\left(y, \frac{\delta_{k}}{2}\right)\right) \subset \widetilde{D_{k}}$ if $\pi^{x}(y) \in D_{k}$. Therefore this integral is

$$
\gtrsim \int_{\pi^{x}(y) \in D_{k}} d \mu(y)=\mu\left(\left\{y: \pi^{x}(y) \in D_{k}\right\}\right) .
$$

3.1. Estimate of Good. With $\mu=\mu_{E, \operatorname{Good}_{k, s}^{x}}$ in Lemma 3.1, it follows that

$$
\operatorname{Good} \lesssim \int\left(\sup _{D_{k} \in \Theta_{k}^{\tau}} \int_{\widetilde{D_{k}}} \pi_{*}^{x}\left(\mu_{E, \text { Good }_{k, s}^{x}}^{\delta_{k}}\right)(\omega) d \omega\right) d \mu_{F}(x) .
$$

Notice for each $D_{k} \in \Theta_{k}^{\tau}, \widetilde{D}_{k}$ can be covered by $\lesssim \delta_{k}^{-\tau}$ caps of radius $\delta_{k}$. Therefore by Cauchy-Schwartz, Good is bounded above by

$$
\begin{aligned}
& \int\left(\sup _{D_{k} \in \Theta_{k}^{\tau}}\left|\widetilde{D_{k}}\right| \int_{\widetilde{D_{k}}}\left|\pi_{*}^{x}\left(\mu_{E, \operatorname{Good}_{k, s}^{x}}^{\delta_{k}}\right)(\omega)\right|^{2} d \omega\right)^{\frac{1}{2}} d \mu_{F}(x) \\
\leqslant & \sup _{D_{k} \in \Theta_{k}^{\tau}}\left|\widetilde{D_{k}}\right|^{\frac{1}{2}} \int\left(\int_{S^{d-1}}\left|\pi_{*}^{x}\left(\mu_{E, \operatorname{Good}_{k, s}^{x}}^{\delta_{k}}\right)(\omega)\right|^{2} d \omega\right)^{\frac{1}{2}} d \mu_{F}(x) \\
\lesssim & \delta_{k}^{\frac{d-1-\tau}{2}}\left(\iint_{S^{d-1}}\left|\pi_{*}^{x}\left(\mu_{E, \operatorname{Good}_{k, s}^{x}}^{\delta_{k}}\right)(\omega)\right|^{2} d \omega d \mu_{F}(x)\right)^{\frac{1}{2}} .
\end{aligned}
$$

Suppose $f \in C_{0}\left(\mathbb{R}^{d}\right)$ (the space of compactly supported continuous functions on $\mathbb{R}^{d}$ ), $\nu$ is a compactly supported Borel measure on $\mathbb{R}^{d}$ and $\operatorname{supp}(f) \cap \operatorname{supp}(\nu)=\emptyset$. Orponen's identity (see Lemma 3.1, [10]) implies that for any $p \in(0, \infty)$,

$$
\int\left\|\pi_{*}^{x} f\right\|_{L^{p}\left(S^{d-1}\right)}^{p} d \nu(x) \approx \int_{S^{d-1}}\left\|\left(P_{\omega}\right)_{*} f\right\|_{L^{p}\left(\left(P_{\omega}\right)_{*}\right)}^{p} d \omega
$$

Unfortunately, since our function $\mu_{E, G_{k o d} \delta_{k, s}^{x}}$ depends on $x$, one cannot apply (3.6) to (3.5) directly. Despite this, the idea of reducing radial projections to orthogonal projections still works. We will work on (3.5) very carefully.

Since $\mu_{E, \operatorname{Good}_{k, s}^{x}}^{\delta_{k}} \in C_{0}^{\infty}, \operatorname{dist}(E, F) \approx 1$, we have for each $\omega \in S^{d-1}, x \in F$,

$$
\pi_{*}^{x}\left(\mu_{E, \operatorname{Good}_{k, s}^{x}}^{\delta_{k}}\right)(\omega) \approx \int_{t \approx 1} \mu_{E, \operatorname{Good}_{k, s}^{x}}^{\delta_{k}}(x+t \omega) d t
$$


Fix $\omega \in S^{d-1}$ and consider

$$
\int\left|\pi_{*}^{x}\left(\mu_{E, \operatorname{Good}_{k, s}^{x}}^{\delta_{k}}\right)(\omega)\right|^{2} d \mu_{F}(x) \approx \int\left|\int_{t \approx 1} \mu_{E, \operatorname{Good}_{k, s}^{x}}^{\delta_{k}}(x+t \omega) d t\right|^{2} d \mu_{F}(x) .
$$

We claim that one can replace $\mu_{F}$ by a compactly supported Borel measure $\mu_{F, \omega}$, without changing the value of (3.7), such that

$$
\left(P_{\omega}\right)_{*} \mu_{F, \omega}\left(B\left(u, \delta_{k}\right)\right) \lesssim \delta_{k}^{s}, \forall u \in \mathbb{R}^{d-1} .
$$

To see this, notice $\mu_{E, \operatorname{Good}_{k, s}^{x}}^{\delta_{k}}$ is supported on the $\delta_{k}$-neighborhood of $\operatorname{Good}_{k, s}^{x}$, so

$$
\int_{t \approx 1} \mu_{E, \operatorname{Good}_{k, s}^{x}}^{\delta_{k}}(x+t \omega) d t
$$

is nontrivial only if there exists $y \in \operatorname{Good}_{k, s}^{x}$ such that the distance between $y$ and the line $\{x+t \omega: t \in \mathbb{R}\}$ is no more than $\delta_{k}$, which is equivalent to $\operatorname{dist}\left(P_{\omega} y, P_{\omega} x\right) \leqslant \delta_{k}$. This means, for each fixed $\omega \in S^{d-1}$, we can restrict $\mu_{F}$ to

$$
\left\{x \in F: \operatorname{dist}\left(P_{\omega}\left(\operatorname{Good}_{k, s}^{x}\right), P_{\omega} x\right) \leqslant \delta_{k}\right\}
$$

without changing the value of (3.7). Denote this restricted measure as $\mu_{F, \omega}$.

Now let us check (3.8). By our construction of $\mu_{F, \omega}$, given any $\delta_{k}$-tube $T^{k}$ of direction $\omega$, we have $\mu_{F, \omega}\left(T^{k}\right) \neq 0$ only if $T^{k} \cap F$ is contained in the $\delta_{k}$-neighborhood of a line $l_{x, y},(x, y) \in \operatorname{Good}_{k, s}$, of direction $\omega$. This implies $T^{k} \subset T_{x, y}^{k}$ and therefore

$$
\mu_{F, \omega}\left(T^{k}\right) \leqslant \mu_{F}\left(T_{x, y}^{k}\right) \leqslant \delta_{k}^{s}
$$

as desired.

Now, with $\mu_{F}$ replaced by $\mu_{F_{k, \omega}}$, (3.7) is reduced to

$$
\begin{aligned}
\int\left|\int_{t \approx 1} \mu_{E, \operatorname{Good}_{k, s}^{x}}^{\delta_{k}}(x+t \omega) d t\right|^{2} d \mu_{F, \omega}(x) & \leqslant \int\left|\int_{t \approx 1} \mu_{E}^{\delta_{k}}(x+t \omega) d t\right|^{2} d \mu_{F, \omega}(x) \\
& \approx \int\left|\left(P_{\omega}\right)_{*} \mu_{E}^{\delta_{k}}\left(P_{\omega} x\right)\right|^{2} d \mu_{F, \omega}(x) \\
& =\int\left|\left(P_{\omega}\right)_{*} \mu_{E}^{\delta_{k}}(u)\right|^{2} d\left(P_{\omega}\right)_{*} \mu_{F, \omega}(u) .
\end{aligned}
$$

We shall show, up to a negligible error term,

$$
\int\left|\left(P_{\omega}\right)_{*} \mu_{E}^{\delta_{k}}(u)\right|^{2} d\left(P_{\omega}\right)_{*} \mu_{F, \omega}(u) \lesssim \delta_{k}^{-d+1+s} \int_{\xi \in \mathbb{R}^{d-1},|\xi| \lesssim \delta_{k}^{-1}} \mid\left(\left.\widehat{\left.P_{\omega}\right)_{*} \mu_{E}}(\xi)\right|^{2} d \xi .\right.
$$

Here the negligible error comes from the fact that $\widehat{\mu_{E}^{\delta_{k}}}(\xi)=\widehat{\mu_{E}}(\xi) \widehat{\phi}\left(\delta_{k} \xi\right)$ is essentially supported on $B\left(0, \delta_{k}^{-1}\right)$. This allows us to replace $\left|\widehat{\mu_{E}^{\delta_{k}}}(\xi)\right|$ by $\left|\widehat{\mu_{E}}(\xi)\right| \chi_{B\left(0, \delta_{k}^{-1}\right)}$ up to a negligible error. 
For any $f \in L^{2}\left(\left(P_{\omega}\right)_{*} \mu_{F, \omega}\right)$, by Fourier inversion we have, up to a negligible error,

$$
\begin{aligned}
& \left(\int\left(P_{\omega}\right)_{*} \mu_{E}^{\delta_{k}}(u) \cdot f(u) d\left(P_{\omega}\right)_{*} \mu_{F, \omega}(u)\right)^{2} \\
\lesssim & \left(\int_{\xi \in \mathbb{R}^{d-1},|\xi| \lesssim \delta_{k}^{-1}}\left|\widehat{\left(P_{\omega}\right)_{*} \mu_{E}}(\xi)\right|\left|f d \widehat{\left(P_{\omega}\right)_{*}} \mu_{F, \omega}(\xi)\right| d \xi\right)^{2} \\
\leqslant & \int_{\xi \in \mathbb{R}^{d-1},|\xi| \lesssim \delta_{k}^{-1}}\left|\widehat{\left({\widehat{P} \omega)_{*}}_{E}\right.}(\xi)\right|^{2} d \xi \cdot \int_{\xi \in \mathbb{R}^{d-1},|\xi| \lesssim \delta_{k}^{-1}}\left|f d \widehat{\left(P_{\omega}\right)_{*}} \mu_{F, \omega}(\xi)\right|^{2} d \xi .
\end{aligned}
$$

Then (3.10) follows from the following lemma and (3.8), with $r=\delta_{k}, m=d-1$.

Lemma 3.2. Given $r>0$, fixed. Let $\mu$ be a Borel measure on $\mathbb{R}^{m}$ such that $\mu(B(u, r)) \lesssim$ $r^{s}$ for any $x \in \mathbb{R}^{m}$. Then

$$
\left.\int_{|\xi| \lesssim r^{-1}}|\widehat{f d \mu}(\xi)|^{2} d \xi \lesssim r^{-m+s}|| f\right|_{L^{2}(\mu)} ^{2} .
$$

Proof. The proof is standard. Let $\psi$ be a positive Schwartz function on $\mathbb{R}^{m}$ whose Fourier transform has bounded support. Then

$$
\int_{|\xi| \lesssim r^{-1}}|\widehat{f d \mu}(\xi)|^{2} d \xi \lesssim \int|\widehat{f d \mu}(\xi)|^{2} \psi(r \xi) d \xi=r^{-m} \int f(x) K(x, y) f(y) d \mu(x) d \mu(y),
$$

where $K(x, y)=\widehat{\psi}\left(\frac{x-y}{r}\right)$. Since

the lemma follows by Shur's test.

$$
\begin{aligned}
\int|K(x, y)| d \mu(x) & \lesssim \int_{|x-y| \lesssim r} d \mu(x) \lesssim r^{s}, \\
\int|K(x, y)| d \mu(y) & \lesssim \int_{|x-y| \lesssim r} d \mu(y) \lesssim r^{s},
\end{aligned}
$$

Due to (3.9), (3.10), we have the following estimate of (3.7): for each fixed $\omega \in S^{d-1}$,

$$
\int\left|\int_{t \approx 1} \mu_{E, \operatorname{Good}_{k, s}^{x}}^{\delta_{k}}(x+t \omega) d t\right|^{2} d \mu_{F}(x) \lesssim \delta_{k}^{-d+1+s} \int_{\xi \in \mathbb{R}^{d-1},|\xi| \lesssim \delta_{k}^{-1}}\left|\widehat{\left(P_{\omega}\right)_{*} \mu_{E}}(\xi)\right|^{2} d \xi .
$$

Plug this into (3.5). It follows that

$$
\begin{aligned}
\text { Good } & \lesssim \delta_{k}^{\frac{d-1-\tau}{2}} \cdot \delta_{k}^{\frac{-d+1+s}{2}} \cdot\left(\int_{S^{d-1}} \int_{\xi \in \mathbb{R}^{d-1},|\xi| \lesssim \delta_{k}^{-1}} \mid\left(\left.\widehat{\left.P_{\omega}\right)_{*} \mu_{E}}(\xi)\right|^{2} d \xi d \omega\right)^{\frac{1}{2}}\right. \\
& =c_{d} \delta_{k}^{\frac{s-\tau}{2}} \cdot\left(\int_{\eta \in \mathbb{R}^{d},|\eta| \lesssim \delta_{k}^{-1}}\left|\widehat{\mu_{E}}(\eta)\right|^{2}|\eta|^{-1} d \eta\right)^{\frac{1}{2}} \\
& \lesssim \delta_{k}^{\frac{s-\tau}{2}} \cdot\left(\sum_{j \leqslant k} \delta_{j} \int_{\eta \in \mathbb{R}^{d},|\eta| \lesssim \delta_{j}^{-1}}\left|\widehat{\mu_{E}}(\eta)\right|^{2} d \eta\right)^{\frac{1}{2}} \\
& \lesssim \delta_{k}^{\frac{-d+1+s_{E}+s-\tau}{2}} \\
& =\delta_{k}^{2 \beta}
\end{aligned}
$$

where the second to the last line follows from Lemma 3.2 and our assumption $s_{E}<d-1$. 
3.2. Estimate of Bad. Now it remains to show

$$
\operatorname{Bad} \lesssim \delta_{k}^{2 \beta}
$$

By the symmetry of $x, y$ in the definition of $\operatorname{Bad}_{k, s}$, we have

$$
\mathbf{B a d}=\int \mu_{E, B a d_{k, s}^{x}}\left(\mathbb{R}^{d}\right) \mu_{F}(x)=\mu_{E} \times \mu_{F}\left(\operatorname{Bad}_{k, s}\right)=\int \mu_{F}\left(\operatorname{Bad}_{k, s}^{y}\right) d \mu_{E}(y) .
$$

Fix $y \in E$. Since $\mu_{F}\left(T_{x, y}^{k}\right)>\delta_{k}^{s}$ for any $x \in F \cap B a d_{k, s}^{y}$, by the $5 r$-covering lemma $F \cap B a d_{k, s}^{y}$ can be covered by $\lesssim \delta_{k}^{-s}$ many $\delta_{k}$-tubes whose central lines pass through $y$. Therefore

$$
\mathbf{B a d} \leqslant \int \sup _{D_{k} \in \Theta_{k}^{s}} \mu_{F}\left(\left\{x: \pi^{y}(x) \in D_{k}\right\}\right) d \mu_{E}(y)
$$

and the whole problem, namely whether $\pi^{x}(E) \geqslant \tau$ for some $x \in F$, is reduced to

$$
\int \sup _{D_{k} \in \Theta_{k}^{s}} \mu_{F}\left(\left\{x: \pi^{y}(x) \in D_{k}\right\}\right) d \mu_{E}(y) \lesssim \delta_{k}^{2 \beta} .
$$

Notice that (3.14) has the same type of (3.2), with $E, F$ swapped and $s$ in place of $\tau$. Therefore (3.14) itself would imply $\operatorname{dim}_{\mathcal{H}} \pi^{y}(F) \geqslant s$ for some $y \in E$. In other words, we start from $\operatorname{dim}_{\mathcal{H}} \pi^{x}(E) \geqslant \tau, x \in F$, and eventually end up with $\operatorname{dim}_{\mathcal{H}} \pi^{y}(F) \geqslant s=$ $d-1+\tau-\operatorname{dim}_{\mathcal{H}} E, y \in E$. This allows us to apply known estimates on $\pi^{y}(F), y \in E$ to obtain improvement on $\pi^{x}(E), x \in F$.

\section{Proof of Theorem 1.1}

It suffices to show (3.14) for any $\tau<s_{E}<\operatorname{dim}_{\mathcal{H}} E$ when $s_{F}>2(d-1)-s_{E}$. By Lemma 3.1 and Hölder, the left hand side of (3.14) is bounded above by

$$
\begin{aligned}
& \int \sup _{D_{k} \in \Theta_{k}^{s}}\left(\int_{\widetilde{D_{k}}} \pi_{*}^{x}\left(\mu_{F}^{\delta_{k}}\right)(\omega) d \omega\right) d \mu_{E}(x) \\
\leqslant & \sup _{D_{k} \in \Theta_{k}^{s}}\left|\widetilde{D_{k}}\right|^{\frac{1}{p^{\prime}}} \int\left(\int_{S^{d-1}}\left|\pi_{*}^{x}\left(\mu_{F}^{\delta_{k}}\right)(\omega)\right|^{p} d \omega\right)^{\frac{1}{p}} d \mu_{E}(x) \\
\lesssim & \delta_{k}^{\frac{d-1-s}{p^{\prime}}}\left(\iint_{S^{d-1}}\left|\pi_{*}^{x}\left(\mu_{F}^{\delta_{k}}\right)(\omega)\right|^{p} d \omega d \mu_{E}(x)\right)^{\frac{1}{p}} .
\end{aligned}
$$

Since $s_{F}>d-1$ and $s_{E}+s_{F}>2(d-1)$, Orponen's estimate (see (3.6) in [10]) in the proof of (1.1) implies that when $p>1$ is small enough (independent in $\delta_{k}$ ),

$$
\iint_{S^{d-1}}\left|\pi_{*}^{x}\left(\mu_{F}^{\delta_{k}}\right)(\omega)\right|^{p} d \omega d \mu_{E}(x) \lesssim I_{s_{E}-\epsilon}\left(\mu_{E}\right)^{\frac{1}{2 p}} \cdot I_{s_{F}-\epsilon}\left(\mu_{F}^{\delta_{k}}\right)^{\frac{1}{2}} \leqslant I_{s_{E}-\epsilon}\left(\mu_{E}\right)^{\frac{1}{2 p}} \cdot I_{s_{F}-\epsilon}\left(\mu_{F}\right)^{\frac{1}{2}}
$$

where $\epsilon>0$ is a fixed small constant. Here the last inequality holds due to the Fourier expression of the energy integral (2.2).

Since $I_{s_{E}-\epsilon}\left(\mu_{E}\right), I_{s_{F}-\epsilon}\left(\mu_{F}\right)<\infty$ and $s=d-1+\tau-s_{E}+4 \beta$, for any $\tau<s_{E}$ we have

$$
\operatorname{Bad} \lesssim \delta_{k}^{2 \beta}
$$

when $\beta>0$ is small enough. This completes the proof of Theorem 1.1 . 


\section{Discussion}

In this section we explain why no new result follows from (1.2), (1.3), (1.5). Recall $\operatorname{dim}_{\mathcal{H}} E \leqslant d-1$ and $s=d-1+\tau-\operatorname{dim}_{\mathcal{H}} E$.

By our framework, to consider if $\operatorname{dim}_{\mathcal{H}} \pi^{x}(E) \geqslant \tau$ for some $x \in F$, it suffices to consider if $\operatorname{dim}_{\mathcal{H}} \pi^{y}(F) \geqslant s$ for some $y \in E$. If we apply (1.2), (1.3), (1.5), the following three sufficient conditions are obtained:

$$
\begin{gathered}
\operatorname{dim}_{\mathcal{H}} E>s+1 ; \\
s<\operatorname{dim}_{\mathcal{H}} F-1 ; \\
s<\frac{\operatorname{dim}_{\mathcal{H}} F}{2}, \text { if } d=2 .
\end{gathered}
$$

The first condition implies

$$
\tau=s+\operatorname{dim}_{\mathcal{H}} E-d+1<2 \operatorname{dim}_{\mathcal{H}} E-d \leqslant \operatorname{dim}_{\mathcal{H}} E-1,
$$

which is already trivial by (1.3).

The second condition implies

$$
\operatorname{dim}_{\mathcal{H}} F>s+1=d+\tau-\operatorname{dim}_{\mathcal{H}} E \geqslant \tau+1
$$

which does not beat (1.2).

The third condition implies

$$
\operatorname{dim}_{\mathcal{H}} F>2+2 \tau-2 \operatorname{dim}_{\mathcal{H}} E=2-\operatorname{dim}_{\mathcal{H}} E+\left(2 \tau-\operatorname{dim}_{\mathcal{H}} E\right) .
$$

When $2 \tau-\operatorname{dim}_{\mathcal{H}} E \leqslant 0$, it does not beat (1.5); when $2 \tau-\operatorname{dim}_{\mathcal{H}} E \geqslant 0$, it does not beat Theorem 1.1. In fact, even if we could obtain $\frac{\operatorname{dim}_{\mathcal{H}} E}{2}+\epsilon_{0}$ in (1.5), no improvement follows for the same reason.

Despite these negative examples, one may still expect our framework to help in the future. For instance, in the sharpness example of (1.1) (see [9]), the exception set lies in a hyperplane. Therefore it is reasonable to expect an improvement if we consider "pins" that do not lie in any hyperplane. If it were true, a corresponding improvement of Theorem 1.1 would follow from our framework, under the extra assumption that $E$ does not lie in any hyperplane.

\section{REFERENCES}

[1] M. Bond, I. Łaba, and J. Zahl. Quantitative visibility estimates for unrectifiable sets in the plane. Trans. Amer. Math. Soc., 368(8):5475-5513, 2016.

[2] L. Guth, A. Iosevich, Y. Ou, and H. Wang. On falconer's distance set problem in the plane. arXiv preprint arXiv.org:1808.09346, 2018.

[3] T. Keleti and P. Shmerkin. New bounds on the dimensions of planar distance sets. https://arxiv.org/abs/1801.08745, 2018.

[4] B. Liu. Hausdorff dimension of pinned distance sets and the $L^{2}$-method. arXiv preprint arXiv:1810.08127, 2018.

[5] J. M. Marstrand. Some fundamental geometrical properties of plane sets of fractional dimensions. Proc. London Math. Soc. (3), 4:257-302, 1954.

[6] P. Mattila. Hausdorff dimension, projections, and the Fourier transform. Publ. Mat., 48(1):3-48, 2004.

[7] P. Mattila. Fourier analysis and Hausdorff dimension, volume 150. Cambridge University Press, 2015.

[8] P. Mattila and T. Orponen. Hausdorff dimension, intersections of projections and exceptional plane sections. Proceedings of the American Mathematical Society, 144(8):3419-3430, 2016. 
[9] T. Orponen. A sharp exceptional set estimate for visibility. Bull. Lond. Math. Soc., 50(1):1-6, 2018.

[10] T. Orponen. On the dimension and smoothness of radial projections. Anal. PDE, 12(5):1273-1294, 2019.

[11] T. Orponen and T. Sahlsten. Radial projections of rectifiable sets. Ann. Acad. Sci. Fenn. Math., 36(2):677-681, 2011.

[12] Y. Peres and W. Schlag. Smoothness of projections, Bernoulli convolutions, and the dimension of exceptions. Duke Math. J., 102(2):193-251, 2000.

[13] P. Shmerkin. On the hausdorff dimension of pinned distance sets. arXiv preprint arXiv:1706.00131, 2017.

[14] K. Simon and B. Solomyak. Visibility for self-similar sets of dimension one in the plane. Real Anal. Exchange, 32(1):67-78, 2006/07.

Department of Mathematics, the Chinese University of Hong Kong, Shatin, N.T., HONG KONG

E-mail address: Bochen.Liu1989@gmail.com 